\title{
A spectral decomposition approach for the mechanical statistical characterization of distributed fiber-reinforced tissues
}

\author{
Marcello Vasta ${ }^{\mathrm{a}}$, Alessio Gizzi ${ }^{\mathrm{b}}$, and Anna Pandolfi ${ }^{\mathrm{c}}$, \\ a Università di Chieti-Pescara, Dipartimento INGEO, \\ Viale Pindaro 42, Pescara, Italy \\ b Campus Bio-Medico University of Rome, Department of Engineering, \\ Via A. del Portillo 21, 00128 Rome, Italy \\ c Politecnico di Milano, Dipartimento di Ingegneria Civile ed Ambientale, \\ Piazza Leonardo da Vinci 32, Milano, Italy
}

\begin{abstract}
We discuss a spectral decomposition formulation for the mechanical statistical characterization of the anisotropic strain energy density of soft hyperelastic materials embedded with distributed fibers. We consider a generalized angular probability density function (PDF) of the reinforcement built upon the local eigenvalue and eigenvector system of the Cauchy-Green deformation tensor. We focus our analysis to material models dependent on the fourth pseudo-invariant of the deformation, $\mathrm{I}_{4}$, and to exponential forms of the fiber strain energy function. Within such a spectral reference system, we derive the closed-form expression of the $\mathrm{PDF}$ for $\mathrm{I}_{4}$ generalizing the multi-value random variable transformation procedure recently developed in Gizzi et al. 2016. Our formulation bypasses the cumbersome extension-contraction switch, commonly adopted for shutting down the contribution of contracted fibers in models based on generalized structure tensors. Accordingly, we identify analytically the support of the fibers in pure extension for significant loading conditions. We can readily compute any statistics of the fourth pseudo-invariant and we can derive the direct definition of the average second Piola-Kirchhoff stress tensor according to the second order approximation.
\end{abstract}

1 Keywords: statistical fiber distribution, spectral decomposition, multivariate, fourth 2 pseudo-invariant, fiber reinforced materials. 
4 In modern applications of biomechanical engineering a paramount role is played by the constitutive modelling of soft tissues, based often on advanced mathematical methods. Notable examples include cardiovascular functioning [1,2], cartilage [3], skin [4], gastric [5] and human cornea [6,7] characterization in the view of surgical planning [8-10], fiber recruitment [11], growth and remodeling theories [12-17], and others. The accurate constitutive modelling of soft tissues is requested by the patient-specific nature and the intrinsic complexity of biological tissues that have to accomplish several functions under various conditions [18]. A reliable constitutive modeling in biomechanics is very challenging and still incomplete $[19,20]$ : it requires to account for highly nonlinear behaviors, distributed inhomogeneities of the mechanical properties, multiple length and time scales, and multiphysics coupling [21].

Robust computational approaches for constitutive models of soft tissues rely on variational formulations, based on the definition of an appropriate strain energy density to describe the reversible behaviors. Advanced models try to address explicitly the hierarchical nature of the material by introducing several length scale parameters [22], or by describing in detail the effects of the microstructure. Constitutive models that account directly for the stochastic spatial distribution of the collagen fibers have originated copiously from the approach first proposed by Lanir [23]. The presence of dispersed fibers confers to the medium a certain degree of anisotropy not easy to be described or quantified, whereas the availability of handy parameters would be highly desirable, especially in numerical applications. The complex, in some cases unaffordable and computationally demanding, description of the microstructure has pushed for approximations based on the homogenization of the microstructure by means of parameters of the collagen distribution (i.e., average and higher order statistics) in the strain energy density [24-26]. Strong homogenization techniques, though, may cancel out the features of the microstructure and compromise the predictive properties of the model at the macroscale [27].

Among countless research papers discussing stochastic models of fiber reinforced materials, only a few contributions have been trying to characterize analytically the probability distribution functions (PDF) by means of statistical descriptors [9,12,28-30]. In particular, the recent work by Gizzi et al. [31] has derived the analytical characterization of the statistics of mechanically significant quantities for soft materials embedded with a stochastic distribution of reinforcing fibers. The present contribution aims at generalizing the results of [31] by considering a spectral decomposition approach [32].

The well established theoretical framework considers hyperelastic materials and departs from the assumption that the anisotropic behavior of the material is described entirely by the fourth pseudo-invariant $\mathrm{I}_{4}$, i. e., the square of the stretch in the direction of the fibers, for both planar and three-dimensional cases [33]. Following [32], a generalized three-dimensional von Mises PDF of the reinforcing fibers is built through the spectral decomposition of the right Cauchy-Green deformation tensor. This expedient allows to 
generalize the bijective random variable transformation recently introduced in [31] and, as a notable novel result, to derive the analytic expression of the $\mathrm{I}_{4}$ 's PDF, denoted $\rho_{\mathrm{I}_{4}}\left(I_{4}\right)$, in a principal reference system.

This generalized framework allows to derive analytically all the statistics of $\mathrm{I}_{4}$. In particular, it is possible to identify uniquely the support, or integration domain, of $\rho_{\mathrm{I}_{4}}\left(I_{4}\right)$, restricted by symmetry properties to half a sphere $\Theta \in[-\pi / 2, \pi / 2], \Phi \in[-\pi / 2, \pi / 2]$. For special cases of loading and mean fiber direction, the formulation recovers well-known results of the literature. Moreover, it allows to identify the entries (integral coefficients) of the generalized averaged structural tensor $\mathbf{H}[24,25]$ and $\mathbb{H}[26]$.

Numerical examples concerning uniaxial tension, biaxial, and simple shear loading can be worked out for different values of the mean direction of collagen fibers $\mathbf{M}$ and PDF concentration parameter $b$. A remarkable result is that the approach is able to provide the analytical expression of the integral coefficients of the selected distribution, known as $\kappa, \hat{\kappa}$ coefficients, for uniaxial loading, generalizing the results existing for transverse isotropic materials $[24,25,31]$. The range of variability of the $\kappa$ coefficient is generalized and shown to reduce to the one reported first in [24] only when the mean direction of the fibers and the loading direction are coincident.

The paper is organized as follows. In Section 2 the models for materials with distributed fibers considered in this study are illustrated and the closed-form PDF for the fourth pseudo-invariant is derived by using a generalized random variable transformation. In Section 3 quantitative comparison between the mechanical response of our novel closedform derivations and the one of alternative models are produced. In Section 4 the results and the limitation of the proposed approach are discussed and commented, and future extensions are proposed.

\section{Methods}

The material models for fiber reinforced tissues proposed here require some mathematical preliminary that is recalled briefly. The following derivation uses the standard notation for finite elasticity kinematics. For the statistical aspects, we comply with the notation X and $x$ to denote an aleatoric variable and its occurrence, respectively. A similar notation is adopted for the fourth pseudo-invariant, indicating the aleatoric variable with $\mathrm{I}_{4}$ and its occurrence with $I_{4}$.

\subsection{Spectral decomposition}

Following [32], we begin by considering the spectral decomposition of the right Cauchy Green deformation tensor $\mathbf{C}=\mathbf{F}^{T} \mathbf{F}$, where $\mathbf{F}=\mathbf{R} \mathbf{U}$ is the deformation gradient, $\mathbf{R}$ the 
rotation tensor, and $\mathbf{U}$ the right stretch tensor:

$$
\mathbf{C}=\lambda_{1}^{2} \mathbf{V}_{1} \otimes \mathbf{V}_{1}+\lambda_{2}^{2} \mathbf{V}_{2} \otimes \mathbf{V}_{2}+\lambda_{3}^{2} \mathbf{V}_{3} \otimes \mathbf{V}_{3},
$$

where $\lambda_{i}$ are the eigenvalues and $\mathbf{V}_{i}$ the eigenvectors of $\mathbf{U}$. With no loss of generality, for an assigned deformation the principal stretches can be ordered as $\lambda_{1} \geq \lambda_{2} \geq \lambda_{3}$. Clearly, the condition $\lambda_{1}=\lambda_{2}=\lambda_{3}$ corresponds to the reference configuration. We deal with incompressible materials, therefore the constraint $\lambda_{1} \lambda_{2} \lambda_{3}=1$ is in force.

In the principal stretch reference system, the generic unit vector $\mathbf{N}$ is described in terms of the spherical Eulerian angles $\Theta \in[0: \pi], \Phi \in[0: 2 \pi]$ as

$$
\mathbf{N}=\sin \Theta \cos \Phi \mathbf{V}_{1}+\sin \Theta \sin \Phi \mathbf{V}_{2}+\cos \Theta \mathbf{V}_{3}
$$

and the fourth pseudo-invariant $I_{4}=\mathbf{F N} \cdot \mathbf{F N}=(\mathbf{N} \otimes \mathbf{N}): \mathbf{C}$ as

$$
\mathrm{I}_{4}(\Theta, \Phi)=\sin ^{2} \Theta\left(\lambda_{1}^{2} \cos ^{2} \Phi+\lambda_{2}^{2} \sin ^{2} \Phi\right)+\lambda_{3}^{2} \cos ^{2} \Theta .
$$

We exclude from our considerations contracted fibers that may violate the stability conditions, and impose the satisfaction of the physical requirement that only the fibers that attain the condition $I_{4} \geq 1$ contribute to the elastic energy and the stress, or, equivalently, that the fibres attaining $I_{4}<1$ are discarded [34].

In most cases, biological tissues are characterized by non planar architecture of reinforcing fibers. The representation of this physical condition requires the adoption of fully three-dimensional (3D) distributions [30]. Moreover, collagen fibers are usually dispersed around a mean referential direction, $\mathbf{M}$ with components $M_{i}$, showing either a rotational symmetry in space or no symmetry at all. In general, $\mathbf{M}$ represents the axis of symmetry of the chosen distribution, e.g. when the concentration parameter $b<0$ (these cases are not considered in the present study). For the applications that we have in mind, in fact, we consider a generic 3D arrangement based on the generalized von Mises distribution [35] $\rho(\mathbf{N})=\rho_{\Theta, \Phi}(\mathbf{N}(\theta, \phi))$ function of the angles $\Theta, \Phi$ as

$$
\rho_{\Theta, \Phi}(\theta, \phi)=4 \sqrt{\frac{b}{2 \pi}} \frac{\exp \left[2 b(\mathbf{N} \cdot \mathbf{M})^{2}\right]}{\operatorname{erfi} \sqrt{2 b}}=\tilde{N}(b) \exp \left[2 b(\mathbf{N} \cdot \mathbf{M})^{2}\right],
$$

where $b$ is the concentration parameter of the distribution and $\tilde{N}(b)$ is a normalization factor dependent on $b$. The symmetry property allows to restrict the study to half sphere $\Theta \in[-\pi / 2, \pi / 2], \Phi \in[-\pi / 2, \pi / 2]$.

For increasing values of the concentration parameter $b$, Fig. 1 illustrates the angular phase plots of the generalized von Mises distribution. Plots refer to three different mean referential directions $\mathbf{M}_{i}$, i. e.,

$$
\mathbf{M}_{1}=[1,0,0], \quad \mathbf{M}_{2}=[0,0,1], \quad \mathbf{M}_{3}=1 / \sqrt{3}[1,1,1],
$$

and show that high values of $b$ concentrate the PDF along particular directions, preserving a non-uniform distribution not implicitly imposed. Changes in the mean referential 


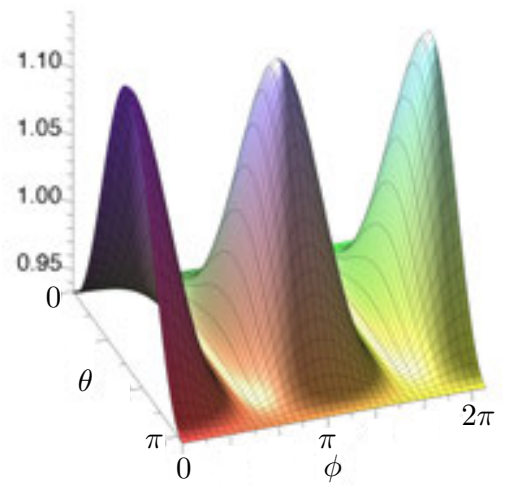

(a) $\mathbf{M}_{1}, b=0.1$

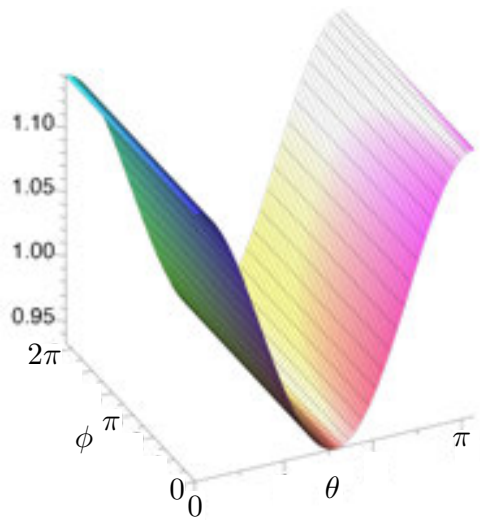

(d) $\mathbf{M}_{2}, b=0.1$

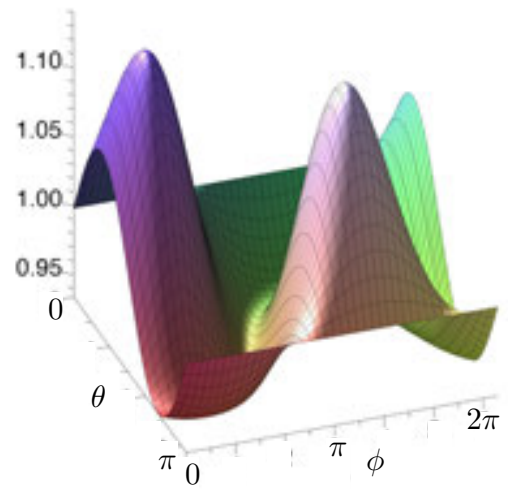

(g) $\mathbf{M}_{3}, b=0.1$

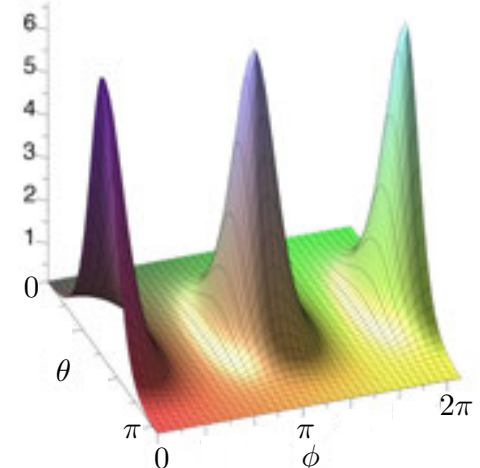

(b) $\mathbf{M}_{1}, b=2$

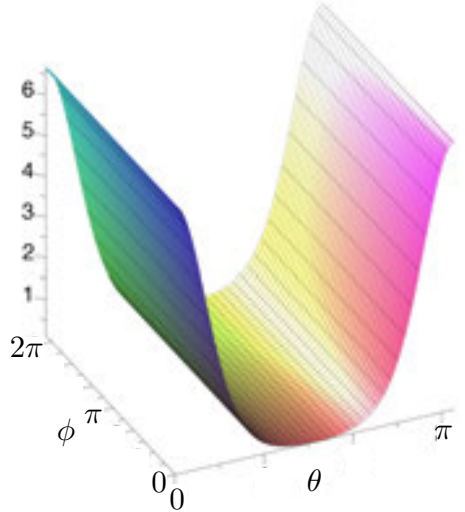

(e) $\mathbf{M}_{2}, b=2$

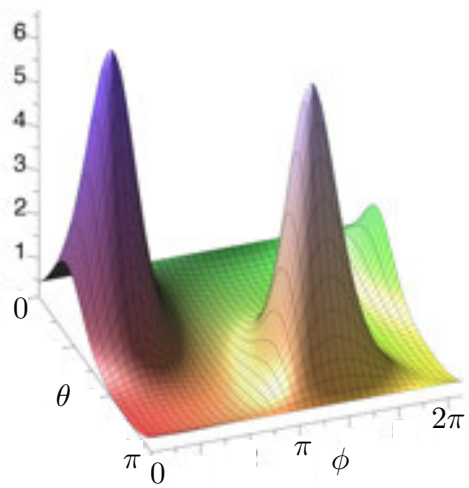

(h) $\mathbf{M}_{3}, b=2$

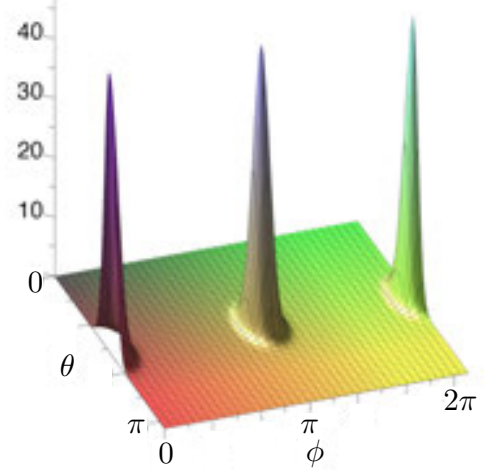

(c) $\mathbf{M}_{1}, b=12$

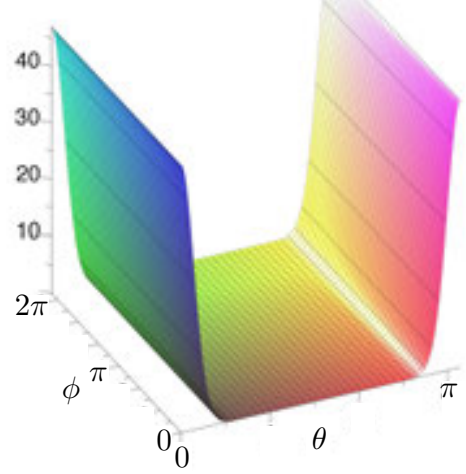

(f) $\mathbf{M}_{2}, b=12$

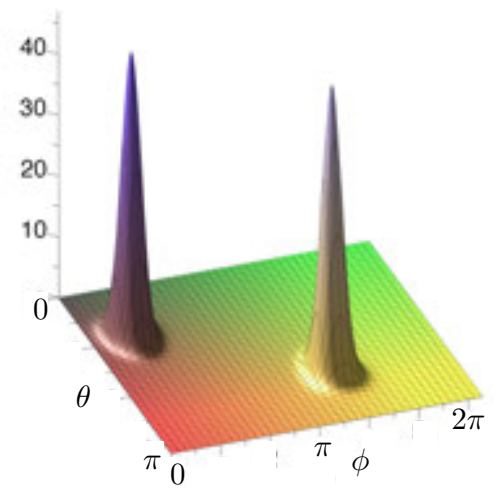

(i) $\mathbf{M}_{3}, b=12$

Fig. 1. Angular phase plots of generalized von Mises PDF, Eq. (4), for three different values of $b$ (columns) and three mean fiber direction $\mathbf{M}_{i}$ (rows).

direction draw different landscapes: multiple periodic PDF for $\mathbf{M}_{1}$, a single $\Theta$-dependent distribution for $\mathbf{M}_{2}$, interfering aperiodic PDFs for $\mathbf{M}_{3}$. Accordingly, the model is expected to be characterized by rather different material constants. 


\subsection{Random variable transformation}

The fourth invariant $I_{4}=I_{4}(\Theta, \Phi)$ is a function of the two random variables $(\Theta, \Phi)$ and inherits from them the random character. Following [31], we can derive the PDF of $\mathrm{I}_{4}$, i. e., $\rho_{\mathrm{I}_{4}}\left(I_{4}\right)$, by considering the analytical expression that links $\mathrm{I}_{4}$ and $\Theta, \Phi$. To this end, we make use of a random transformation procedure, cf. [36], and introduce a new random variable $\mathrm{W}$ with occurrence $w$ as

$$
\left\{\begin{array}{l}
\mathrm{I}_{4}=\mathrm{I}_{4}(\Theta, \Phi) \\
\mathrm{W}=\Phi
\end{array} .\right.
$$

The two-dimensional support of the two aleatoric variables $\left(\mathrm{I}_{4}, \mathrm{~W}\right)$ is defined as $\mathcal{F}=$ $\left\{\left[1, \lambda_{1}^{2}\right] \cup[-\pi / 2, \pi / 2]\right\}$.

Remark Note that the lowerbound for the random variable $I_{4}$ is defined by the noncontracted condition $I_{4}=1$ and not by the minimum eigenvalue $\lambda_{3}^{2}<1$. The upperbound of $\mathrm{I}_{4}$ is defined instead by the maximum eigenvalue $\lambda_{1}^{2}=\max \left\{\mathrm{I}_{4}(\Theta, \Phi)\right\}$ [37].

Depending on the loading, the inverse mapping Eq. (6) may be defined univocally on the whole domain $\mathcal{F}$ (bijective transformation)

$$
\left\{\begin{array}{l}
\Theta=\Theta\left(\mathrm{I}_{4}, \mathrm{~W}\right) \\
\Phi=\mathrm{W}
\end{array} \in \mathcal{F},\right.
$$

or on $m$ subdomains $\sum_{i} \subset\left\{\left[1, \lambda_{1}^{2}\right] \cup[-\pi / 2, \pi / 2]\right\}$ with $i=1 \ldots m$ such that $\bigcup_{i=1}^{m} \sum_{i}=\mathcal{F}$ (multivalued transformation)

$$
\left\{\begin{array}{l}
\Theta=\Theta_{i}\left(\mathrm{I}_{4}, \mathrm{~W}\right) \\
\Phi=\mathrm{W}
\end{array} \in \Sigma_{i} .\right.
$$

We discuss the two cases separately.

\subsubsection{Bijective Transformation}

When the inverse mapping is bijective (2.2), we begin by evaluating the Jacobian of the transformation, which reduces to the derivative of $I_{4}$ with respect to $\theta$ :

$$
[\mathbf{J}]=\left[\begin{array}{ll}
\frac{\partial I_{4}}{\partial \theta} & \frac{\partial I_{4}}{\partial \phi} \\
\frac{\partial w}{\partial \theta} & \frac{\partial w}{\partial \phi}
\end{array}\right]=\left[\begin{array}{cc}
\frac{\partial I_{4}}{\partial \theta} & \frac{\partial I_{4}}{\partial \phi} \\
0 & 1
\end{array}\right], \quad \operatorname{det} \mathbf{J}=\frac{\partial I_{4}}{\partial \theta}
$$


The joint PDF $\rho_{\mathrm{I}_{4}, \mathrm{~W}}\left(I_{4}, w\right)$ of the two transformed random variables $\left(\mathrm{I}_{4}, \mathrm{~W}\right)$ is related to the joint PDF of the two random angle variables $\rho_{\Theta, \Phi}(\theta, \phi)$ as [31]

$$
\rho_{\mathrm{I}_{4}, \mathrm{~W}}\left(I_{4}, w\right)=\left.\frac{\rho_{\Theta, \Phi}(\theta, \phi) \sin \theta}{\operatorname{det} \mathbf{J}}\right|_{\substack{\theta=\theta\left(I_{4}, w\right) \\ \phi=w}}=\left.\rho_{\Theta, \Phi}(\theta, \phi) \sin \theta\left(\frac{\partial I_{4}}{\partial \theta}\right)^{-1}\right|_{\substack{\theta=\theta\left(I_{4}, w\right) \\ \phi=w}}
$$

thus the $\mathrm{PDF}$ of $\mathrm{I}_{4}$ can be evaluated as the marginal density of $\rho_{\mathrm{I}_{4}, \mathrm{~W}}\left(I_{4}, w\right)$, i. e.,

$$
\rho_{\mathrm{I}_{4}}\left(I_{4}\right)=\left.\int_{-\pi / 2}^{\pi / 2} \rho_{\Theta, \Phi}(\theta, \phi) \sin \theta\left(\frac{\partial I_{4}}{\partial \theta}\right)^{-1}\right|_{\substack{\theta=\theta\left(I_{4}, w\right) \\ \phi=w}} d w
$$

For the general case in a 3D setting, it is possible to obtain the inverse mapping by using Eq. (3)

$$
\left\{\begin{array}{l}
\sin ^{2} \Theta=\frac{\mathrm{I}_{4}-\lambda_{3}^{2}}{\lambda_{1}^{2} \cos ^{2} \Phi+\lambda_{2}^{2} \sin ^{2} \Phi-\lambda_{3}^{2}} \\
\cos ^{2} \Theta=\frac{\lambda_{1}^{2} \cos ^{2} \Phi+\lambda_{2}^{2} \sin ^{2} \Phi-\mathrm{I}_{4}}{\lambda_{1}^{2} \cos ^{2} \Phi+\lambda_{2}^{2} \sin ^{2} \Phi-\lambda_{3}^{2}}
\end{array}\right.
$$

thus

$$
\Theta= \pm \arcsin \sqrt{\frac{\mathrm{I}_{4}-\lambda_{3}^{2}}{\lambda_{1}^{2} \cos ^{2} \Phi+\lambda_{2}^{2} \sin ^{2} \Phi-\lambda_{3}^{2}}} .
$$

Note that the inverse mapping will be bijective or multivalued according to the values assumed by the eigenvalues $\lambda_{i}$. However, in this section we are considering only bijective transformations. To obtain $\rho_{\mathrm{I}_{4}}\left(I_{4}\right)$, we manipulate Eq. (11) as

$$
\left.\sin \theta\left(\frac{\partial I_{4}}{\partial \theta}\right)^{-1}\right|_{\substack{\theta=\theta\left(I_{4}, w\right) \\ \phi=w}}=\frac{1}{2 \sqrt{\left(a-\lambda_{3}^{2}\right)\left(a-I_{4}\right)}}
$$

where $a=\lambda_{1}^{2} \cos ^{2} w+\lambda_{2}^{2} \sin ^{2} w$. The composition of $\mathbf{N}$ with $\mathbf{M}$, adopted in the generalized von Mises distribution (4), allows to write

$$
\mathbf{N} \cdot \mathbf{M}=\sin \Theta\left(M_{1} \cos \Phi+M_{2} \sin \Phi\right)+M_{3} \cos \Theta,
$$

and $\rho_{\mathrm{I}_{4}}\left(I_{4}\right)$ derives as

$$
\rho_{\mathrm{I}_{4}}\left(I_{4}\right)=\tilde{N}(b) \int_{-\pi / 2}^{\pi / 2} \frac{\exp \left\{2 b\left[\sqrt{\frac{I_{4}-\lambda_{3}^{2}}{a-\lambda_{3}^{2}}}\left(M_{1} \cos w+M_{2} \sin w\right)+M_{3} \sqrt{\frac{a-I_{4}}{a-\lambda_{3}^{2}}}\right]^{2}\right\}}{\sqrt{\left(a-\lambda_{3}^{2}\right)\left(a-I_{4}\right)}} d w
$$

Eq. (16) is a novel result obtained through the spectral decomposition, and it generalizes the derivation obtained in [31]. 
157

\subsubsection{Multivalued Transformation}

When the inverse mapping is multivalued (2.2) it is necessary in general to define $m$ subdomains where bijectivity is satisfied. The joint probability density $\rho_{\mathrm{I}_{4}, \mathrm{~W}}\left(I_{4}, w\right)$ of the transformed random variables $\left(\mathrm{I}_{4}, \mathrm{~W}\right)$ is related to the joint probability density $\rho_{\Theta, \Phi}(\theta, \phi)$ as

$$
\rho_{\mathrm{I}_{4}, \mathrm{~W}}\left(I_{4}, w\right)=\left.\sum_{i=1}^{m} \frac{\rho_{\Theta, \Phi}(\theta, \phi) \sin \theta}{\operatorname{det} \mathbf{J}_{i}}\right|_{\substack{\theta=\theta_{i}\left(I_{4}, w\right) \\ \phi=w}}=\left.\sum_{i=1}^{m} \rho_{\Theta, \Phi}(\theta, \phi) \sin \theta\left(\frac{\partial I_{4}}{\partial \theta}\right)^{-1}\right|_{\substack{\theta=\theta_{i}\left(I_{4}, w\right) \\ \phi=w}}
$$

and the PDF density of $\mathrm{I}_{4}$ can be evaluated as

$$
\rho_{\mathrm{I}_{4}}\left(I_{4}\right)=\tilde{N}(b) \sum_{i=1}^{m} \int_{-w_{i}\left(I_{4}\right)}^{w_{i}\left(I_{4}\right)} \frac{\exp \left\{2 b\left[\sqrt{\frac{I_{4}-\lambda_{3}^{2}}{a-\lambda_{3}^{2}}}\left(M_{1} \cos w+M_{2} \sin w\right)+M_{3} \sqrt{\frac{a-I_{4}}{a-\lambda_{3}^{2}}}\right]^{2}\right\}}{\sqrt{\left(a-\lambda_{3}^{2}\right)\left(a-I_{4}\right)}} d w
$$

where the limits of the integral, required for the saturation of $w$, depend on the occurrence of the aleatoric variable $\mathrm{I}_{4}$ itself.

Remark. The integration intervals $\left[-w_{i}\left(I_{4}\right), w_{i}\left(I_{4}\right)\right]$ are determined from the properties of the arcsin function (see Eq. (13)), thus each of them is identified on the basis of the value of the fourth invariant. We provide a detailed analysis on this point in the next section. Note that to build $\rho_{\mathrm{I}_{4}}\left(I_{4}\right)$ it is necessary to perform a numerical integration for each deformation state.

\subsubsection{Determination of the integral bounds of a multivariate mapping}

From the mapping $\Theta=\Theta\left(\mathrm{I}_{4}, \lambda_{i}\right)$

$$
\Theta= \pm \arcsin \sqrt{\frac{\mathrm{I}_{4}-\lambda_{3}^{2}}{\lambda_{1}^{2} \cos ^{2} \Phi+\lambda_{2}^{2} \sin ^{2} \Phi-\lambda_{3}^{2}}}
$$

e obtain the range of variability of the arcsin argument

$$
-1 \leq \sqrt{\frac{\mathrm{I}_{4}-\lambda_{3}^{2}}{\lambda_{1}^{2}-\lambda_{3}^{2}-\left(\lambda_{1}^{2}-\lambda_{2}^{2}\right) \sin ^{2} \Phi}} \leq 1
$$

which, in the real domain, leads to the inequalities

$$
\left\{\begin{array}{l}
\frac{\mathrm{I}_{4}-\lambda_{3}^{2}}{\lambda_{1}^{2}-\lambda_{3}^{2}-\left(\lambda_{1}^{2}-\lambda_{2}^{2}\right) \sin ^{2} \Phi} \geq 0 \\
\frac{\mathrm{I}_{4}-\lambda_{3}^{2}}{\lambda_{1}^{2}-\lambda_{3}^{2}-\left(\lambda_{1}^{2}-\lambda_{2}^{2}\right) \sin ^{2} \Phi} \leq 1
\end{array}\right.
$$


Using Eq. (8) trite algebraic calculations give

$$
\left\{\begin{array}{l}
\mathrm{I}_{4} \geq \lambda_{3}^{2} \cap \sin ^{2} w<\frac{\lambda_{1}^{2}-\lambda_{3}^{2}}{\lambda_{1}^{2}-\lambda_{2}^{2}} \cap \sin ^{2} w<\frac{\lambda_{1}^{2}-\mathrm{I}_{4}}{\lambda_{1}^{2}-\lambda_{2}^{2}} \\
\mathrm{I}_{4} \leq \lambda_{3}^{2} \cap \sin ^{2} w>\frac{\lambda_{1}^{2}-\lambda_{3}^{2}}{\lambda_{1}^{2}-\lambda_{2}^{2}} \cap \sin ^{2} w<\frac{\lambda_{1}^{2}-\mathrm{I}_{4}}{\lambda_{1}^{2}-\lambda_{2}^{2}}
\end{array}\right.
$$

where the equality sign has been removed to exclude null denominator. Condition $(21)_{2}$ holds only for $I_{4}=1$ and corresponds to a degenerate case with no deformation.

By imposing the strict inequality for the eigenvalues, $\lambda_{1}>\lambda_{2}>\lambda_{3}$, and using the upper and lower bounds of $\mathrm{I}_{4}$, inequality $(21)_{1}$ reduces to

$$
\sin ^{2} w \leq \frac{\lambda_{1}^{2}-\mathrm{I}_{4}}{\lambda_{1}^{2}-\lambda_{2}^{2}}, \quad \text { if } \quad \lambda_{1} \neq \lambda_{2}
$$

valid on the entire support of $\mathrm{I}_{4}$. Equation (22) allows for the direct identification of the integral bounds in Eq. (18) as

$$
-\alpha\left(\mathrm{I}_{4}\right)<w<\alpha\left(\mathrm{I}_{4}\right)
$$

where $\alpha\left(I_{4}\right)=\arcsin \sqrt{\frac{\lambda_{1}^{2}-\mathrm{I}_{4}}{\lambda_{1}^{2}-\lambda_{2}^{2}}}$. It is possible to prove that the same result is recovered by imposing the condition of positive square roots in Eq. (18)

$$
\left(a-\lambda_{3}^{2}\right)\left(a-\mathrm{I}_{4}\right)>0 \text {. }
$$

Remark. If the condition $\lambda_{1}=\lambda_{2}$ is satisfied, the dependence of Eq. (20) on $\Phi$ and W is excluded and $w$ can be integrated over the whole domain.

Remark. The arcsin function leads to (i) continuous mapping (monotonicity), (ii) symmetric intervals.

On the basis of the previous considerations, $\rho_{\mathrm{I}_{4}}\left(I_{4}\right)$ can be evaluated always by means of the relationship

$$
\rho_{\mathrm{I}_{4}}\left(I_{4}\right)=\tilde{N}(b) \int_{-\alpha\left(I_{4}\right)}^{\alpha\left(I_{4}\right)} \frac{\exp \left\{2 b\left[\sqrt{\frac{I_{4}-\lambda_{3}^{2}}{a-\lambda_{3}^{2}}}\left(M_{1} \cos w+M_{2} \sin w\right)+M_{3} \sqrt{\frac{a-I_{4}}{a-\lambda_{3}^{2}}}\right]^{2}\right\}}{\sqrt{\left(a-\lambda_{3}^{2}\right)\left(a-I_{4}\right)}} d w .
$$

The integration intervals identified in Eq. (23) generalize the calculation proposed in [31] and provide a unique set of inequalities, independent of the direction of the local loading, which can be applied readily to any deformation state. We will provide some example in the following. 


\subsection{Closed-form statistics of $I_{4}$}

\section{Numerical Analysis}

Note that the support of $\mathrm{I}_{4}$ changes according to the loading pattern. In the following we highlight the uniaxial, biaxial and shear cases.

The explicit expression of the $\rho_{\mathrm{I}_{4}}\left(I_{4}\right) \mathrm{PDF}$ in Eq. (24) allows to obtain all the statistics of $\mathrm{I}_{4}$. The average and variance, in particular, are given by the relations

$$
\begin{gathered}
\left\langle\mathrm{I}_{4}\right\rangle=I_{4}^{*}=\int_{1}^{\lambda_{1}^{2}} I_{4} \rho_{\mathrm{I}_{4}}\left(I_{4}\right) d I_{4}, \\
\left\langle\left(\mathrm{I}_{4}-I_{4}^{*}\right)^{2}\right\rangle=\sigma_{I_{4}}^{2}=\int_{1}^{\lambda_{1}^{2}}\left(\mathrm{I}_{4}-I_{4}^{*}\right)^{2} \rho_{\mathrm{I}_{4}}\left(I_{4}\right) d I_{4},
\end{gathered}
$$

that can be used directly to provide the stress tensor in computational applications, cf. $[7,11,26,33]$. In Appendix A we provide a simple example of usage of $I_{4}^{*}$ and $\sigma_{I_{4}}^{2}$.

We investigate isochoric deformations, based on the incompressibility assumption, under uniaxial, biaxial and shear loading patterns for three values of the concentration parameter, $b_{1}=0.1, b_{2}=2$, and $b_{3}=10$. The parameters are chosen to describe isotropic, dispersed and aligned distributions of the fiber reinforcement. We consider the three mean orientations of the distribution in Eq. (5), exploring the aspects of $\rho_{\mathrm{I}_{4}}\left(I_{4}\right)$ for all the relevant combinations of mean direction, concentration parameter, and loading pattern. We omit the results for the direction $[0,1,0]^{T}$, equivalent to $\mathbf{M}_{1}$.

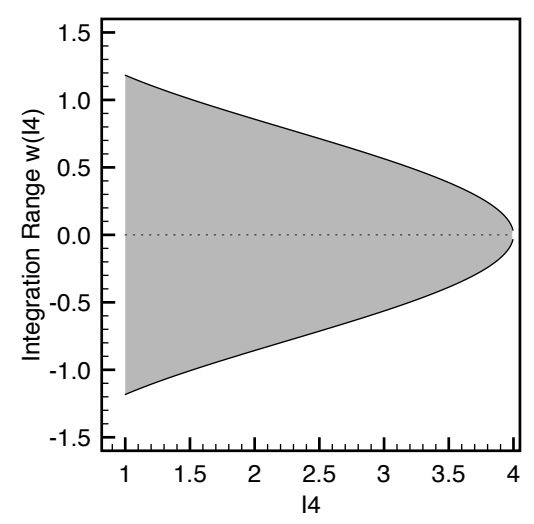

(a) Uniaxial $(\lambda=2)$

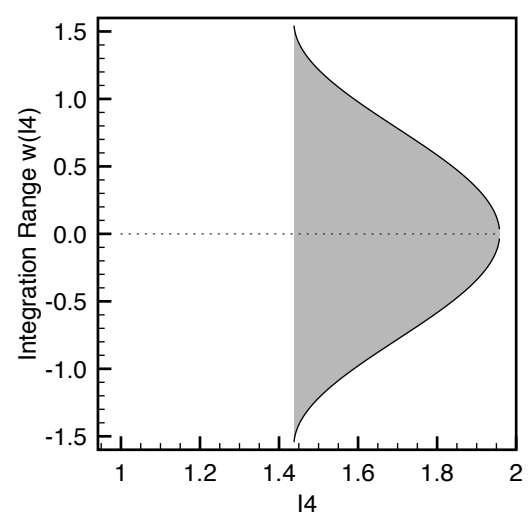

(b) Biaxial $\left(\lambda_{1}=1.4, \lambda_{2}=1.2\right)$

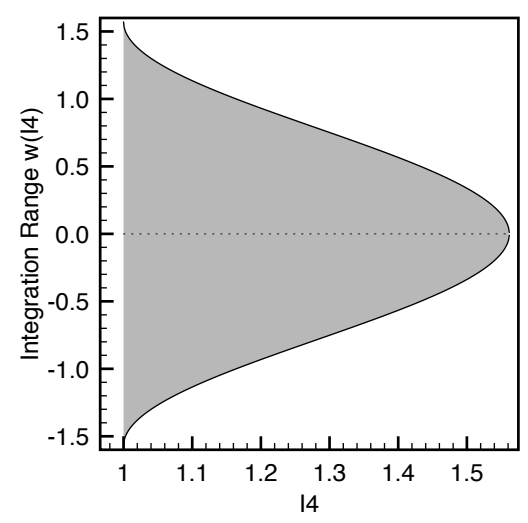

(c) Shear $\left(\lambda_{1}=1.25\right)$

Fig. 2. Implicit plots of the integral condition (26) for (a) uniaxial, (b) biaxial and (c) shear loading. The gray area visualize the contour of the admissible integration ranges. 


\subsection{Uniaxial Loading}

We start from the generalized uniaxial loading with principal stretches:

$$
\lambda_{1}=\lambda=2, \quad \lambda_{2}=\frac{1}{\sqrt{\lambda}}, \quad \lambda_{3}=\frac{1}{\sqrt{\lambda}},
$$

for which condition (23) becomes:

$$
-\arcsin \frac{1}{7} \sqrt{-14 I_{4}+56}<w\left(I_{4}\right)<\arcsin \frac{1}{7} \sqrt{-14 I_{4}+56} .
$$

The bounded region is shown in Fig. 2(a) in the form of an implicit plot of the bound condition $w=w\left(I_{4}\right)$. The internal grey area represents the integration domain where $\rho_{\mathrm{I}_{4}}\left(I_{4}\right)$ has to be computed. In particular, the lower bound coincides with the stability limit, $I_{4}=1$, while the upper bound is given by $I_{4}=\lambda_{1}^{2}$.

Figs. 3(a-c) visualize the results of the numerical integration of Eq. (24), where $w$ is saturated according to the integral domain defined in Eq. (26). Plots compare the computed value of $\rho_{\mathrm{I}_{4}}\left(I_{4}\right)$ for the three fiber orientations (5) and three values of the concentration parameter $b$. Note that $\rho_{\mathrm{I}_{4}}\left(I_{4}\right)$ reveals opposite peak for $\mathbf{M}_{1}$ and $\mathbf{M}_{2}$, whereas more complex nonlinear trends are observed for $\mathbf{M}_{3}$.

\subsection{Biaxial Loading}

Next we consider the generic biaxial loading case with stretches

$$
\lambda_{1}=1.4, \quad \lambda_{2}=1.2, \quad \lambda_{3}=\frac{1}{\lambda_{1} \lambda_{2}}=0.6,
$$

for which condition (23) reduces to

$$
-\arcsin \sqrt{-1.92 I_{4}+3.76}<w\left(I_{4}\right)<\arcsin \sqrt{-1.92 I_{4}+3.76} .
$$

Fig. 2(b) visualizes the implicit plot of the bound conditions $w=w\left(I_{4}\right)$. Unlike the uniaxial case, here the whole integration range of $w$ is considered, i. e. $\pm \pi / 2$. In addition the support of $\mathrm{I}_{4}$ presents lower and upper bounds equal to $\lambda_{2}^{2}=1.44$ and $\lambda_{1}^{2}=1.96$, respectively, thus excluding both $\lambda_{3}^{2}<1$ and $I_{4}=1$.

Figs. 3(d-f) visualizes the results of the numerical integration of Eq. (24) for the three fiber orientation (5) and for three values of the concentration parameter $b$. The behaviors for the biaxial loading (with a marked difference between the two stretches) are rather similar behaviors to ones corresponding to the uniaxial loading, except for the orientation $\mathbf{M}_{3}$ that reveals a smoother behavior. 


\subsection{Shear Loading}

For the case of simple shear we assign the following principal stretches as

$$
\lambda_{1}=\lambda=1.25, \quad \lambda_{2}=1, \quad \lambda_{3}=\frac{1}{\lambda}=0.8,
$$

for which condition (23) becomes

$$
-\arcsin \sqrt{-1.78 I_{4}+2.78}<w\left(I_{4}\right)<\arcsin \sqrt{-1.78 I_{4}+2.78} .
$$

Fig. 2(c) visualizes condition (27), which results as a combination of uniaxial and biaxial conditions since it is expressed in the principal stretch reference system. In particular, the $w\left(\mathrm{I}_{4}\right)$ spans over the whole integration range with lower bound $I_{4}=1$ and upper bound $I_{4}=\lambda_{1}^{2}$. Fig. 3(g-i) visualizes the results of the numerical integration of Eq. (24) for the three fiber orientation (5) and for three values of the concentration parameter. Also the resulting $\rho_{\mathrm{I}_{4}}\left(I_{4}\right)$ mixes the features of uniaxial and biaxial loading.

\section{Conclusions}

The aim of this work is to derive the probability distribution function of the fourth pseudoinvariant $\mathrm{I}_{4}$ of the deformation gradient by means of the random variable procedure introduced in [31] and within the general spectral decomposition framework [32].

The methodology is general and applicable to soft biological collagen-reinforced tissues characterized by the local presence of either three-dimensional or planar distributions of the collagen fibers [33], that can be found in several organs, e.g., the human cornea.

The framework allows to derive in analytical form the statistical structure of $I_{4}$, inherited from the probabilistic distribution of the collagen orientation, and to apply the stability condition to exclude contracted fibers. The results provide a sound theoretical basis for the development of new material models, apt to describe the behavior of biological tissues and suitable to be used in efficient computational algorithms in the view of patient-specific applications in biomechanics. The interest in the development of these models derives mainly from the need of extremely reliable models to be used in the prediction of the outcomes of refractive surgery [7].

Future extensions of the present work will deal with compressible material models, such to avoid restrictions of the principal stretches relations. The inclusion of inelastic phenomena associated to the fiber distribution, e. g. growth, remodeling and active dynamics, can be straightforwardly incorporated holding the spectral statistical framework that can be further generalized to multiphasic heterogeneous media. 


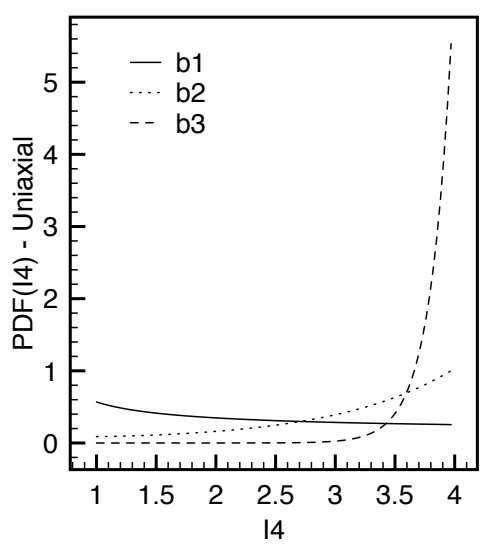

(a) $\mathbf{M}_{1}$

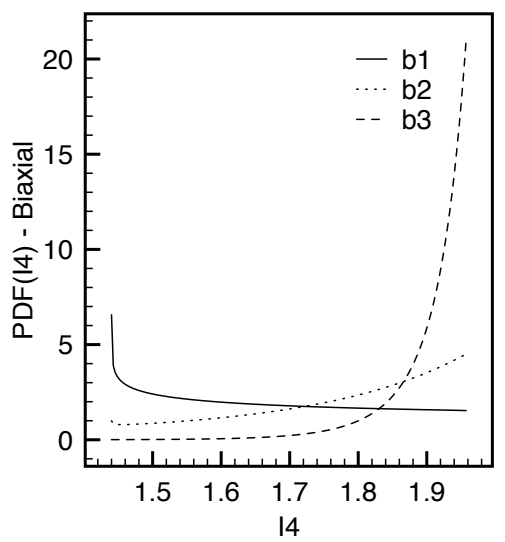

(d) $\mathbf{M}_{1}$

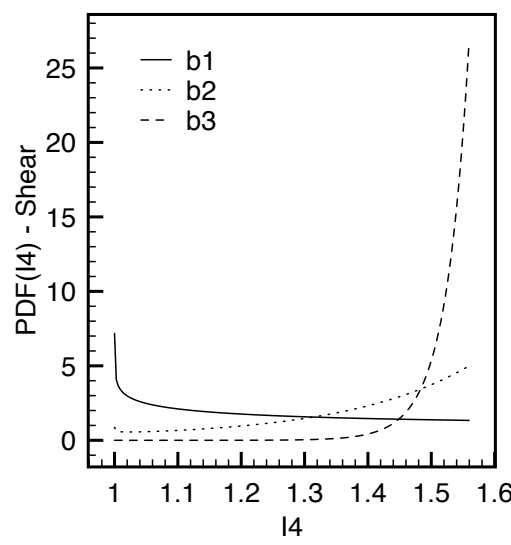

(g) $\mathbf{M}_{1}$

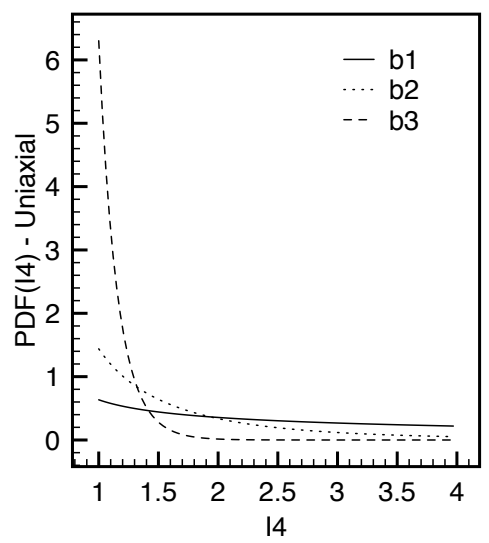

(b) $\mathbf{M}_{2}$

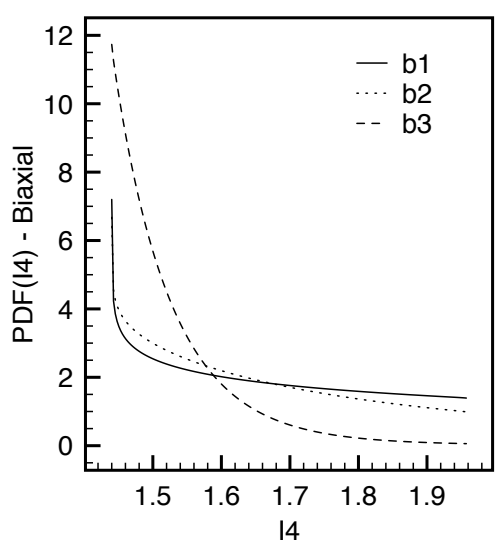

(e) $\mathbf{M}_{2}$

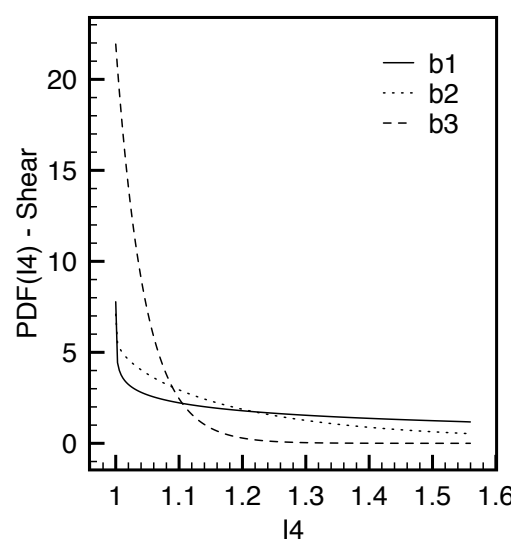

(h) $\mathbf{M}_{2}$

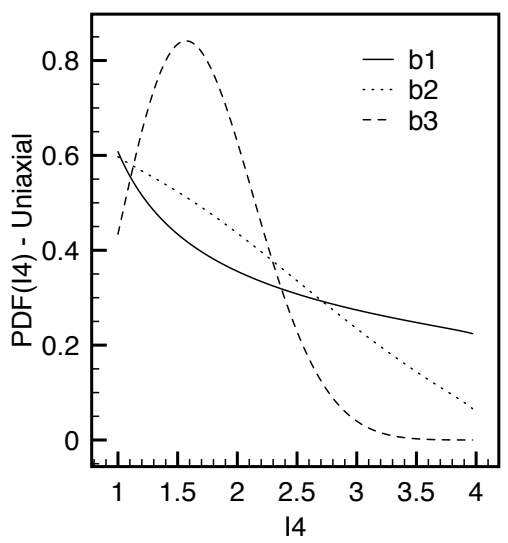

(c) $\mathbf{M}_{3}$

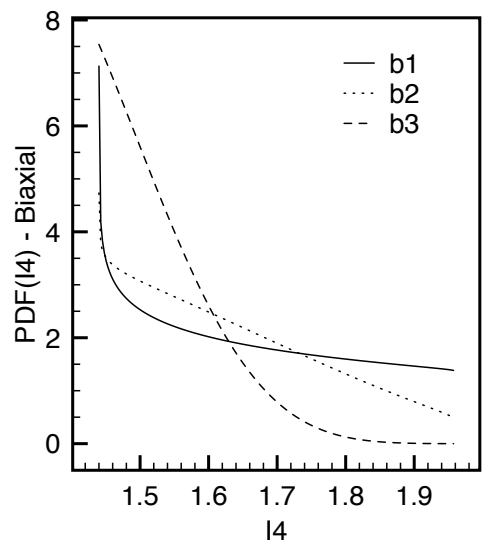

(f) $\mathbf{M}_{3}$

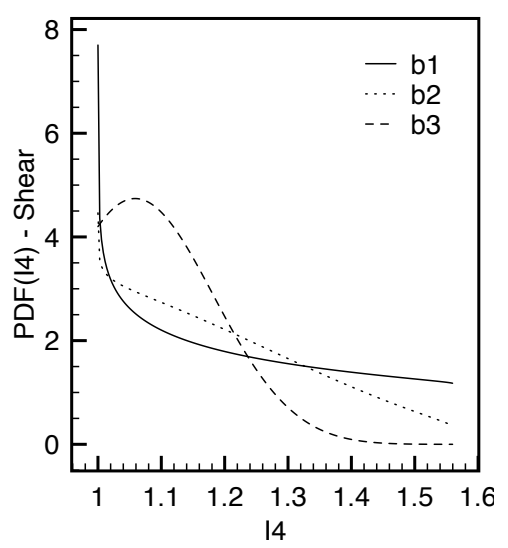

(i) $\mathbf{M}_{3}$

Fig. 3. PDFs of the fourth pseudo-invariant, $\rho_{\mathrm{I}_{4}}\left(I_{4}\right)$, under different loading condition, visualized for three mean directions of the fiber distribution, $\mathbf{M}_{1}, \mathbf{M}_{2}$, and $\mathbf{M}_{3}$, and for three values of the dispersion parameter $b$, corresponding to $b_{1}=0.1$, isotropic distribution, $b_{2}=2$, highly dispersed distribution, and $b_{3}=10$ transversely anisotropic distribution (cf. Eq. (24)). (a-c) Uniaxial loading. (d-f) Biaxial loading. (g-i) Shear loading. 


\section{Acknowledgements}

Authors wish to thank the support from the Italian National Group of Mathematical Physics (GNFM-INdAM). Authors acknowledge useful discussions with G.A. Holzapfel and K. Li.

\section{References}

[1] A. Tsamis, J. T. Krawiec, and D. A. Vorp. Elastin and collagen fibre microstructure of the human aorta in ageing and disease: a review. Journal of the Royal Society Interface, 10:20121004, 2013.

[2] A. Bianchi, E. Monaldo, A. Gizzi, M. Marino, S. Filippi, and G. Vairo. A FSI computational framework for vascular physiopathology: A novel flow-tissue multiscale strategy. Medical Engineering and Physics, 47:25-37, 2017.

[3] S. Federico, A. Grillo, G. La Rosa, G. Giaquinta, and W. Herzog. A transversely isotropic, transversely homogeneous microstructural-statistical model of articular cartilage. Journal of Biomechanics, 103:2008-2018, 2005.

[4] A. Ni Annaidh, K. Bruyere, M. Destrade, M. D. Gilchrist, C. Maurini, M. Ottenio, and G. Saccomandi. Automated estimation of collagen fibre dispersion in the dermis and its contribution to the anisotropic behaviour of skin. Annals of Biomedical Engineering, 40:1666-1678, 2012.

[5] R. C. Aydin, S. Brandstaeter, F. A. Breau, M. Steigenberger, R. P. Marcus, K. Nikolaou, M. Notohamiprodjo, and C. J. Cyron. Experimental characterization of the biaxial mechanical properties of porcine gastric tissue. Journal of the Mechanical Behavior of Biomedical Materials, 74:499-506, 2017.

[6] P. Sánchez, K. Moutsouris, and A. Pandolfi. Biomechanical and optical behavior of human corneas before and after photorefractive keratectomy. Journal of Cataract $\mathcal{E}$ Refractive Surgery, 40:905-917, 2014.

[7] A. Montanino, A. Gizzi, M. Vasta, M. Angelillo, and A. Pandolfi. Modeling the biomechanics of the human cornea accounting for local variations of the collagen fibril architecture. ZAMM, (10.1002/zamm.201700293), 2018.

[8] A. Gizzi, C. Cherubini, N. Pomella, P. Persichetti, M. Vasta, and S. Filippi. Computational modeling and stress analysis of columellar biomechanics. Journal of the Mechanical Behavior of Biomedical Materials, 15:46-58, 2012.

[9] S. Polzer and T. C. Gasser. Biomechanical rupture risk assessment of abdominal aortic aneurysms based on a novel probabilistic rupture risk index. Journal of The Royal Society Interface, 12:20150852, 2015. 
[10] P. Simone, C. Carusi, S. Segreto, R. Iannuzzi, S. Buscaglione, A. Gizzi, S. Giannitelli, A. Rainer, S. Filippi, and P. Persichetti. Postbariatric brachioplasty with posteromedial scar: Physical model, technical refinements, and clinical outcomes. Plastic and Reconstructive Surgery, 141:344-353, 2018.

[11] A. Gizzi, M. Vasta, and A. Pandolfi. Modeling collagen recruitment in hyperelastic biomaterial models with statistical distribution of the fiber orientation. International Journal of Engineering Science, 78:48-60, 2014.

[12] J. F. Rodríquez, F. Cacho, J. A. Bea, and M. Doblaré. A stochastic-structurally based three dimensional finite-strain damage model for fibrous soft tissue. Journal of the Mechanics and Physics of Solids, 44:864-886, 2006.

[13] V. Alastrué, J. F. Rodríguez, B. Calvo, and M. Doblaré. Structural damage models for fibrous biological soft tissues. International Journal of Solids and Structures, 44:5894-5911, 2007.

[14] A. Grillo, G. Wittum, A. Tomic, and S. Federico. Remodelling in statistically oriented fibrereinforced materials and biological tissues. Mathematics and Mechanics of Solids, 20:1107$1129,2015$.

[15] C. J. Cyron, R. C. Aydin, and J. D. Humphrey. A homogenized constrained mixture (and mechanical analog) model for growth and remodeling of soft tissue. Biomechanical Modeling in Mechanobiology, 15:1389-1403, 2016.

[16] A. Grillo, M. Carfagna, and S. Federico. An allen-cahn approach to the remodelling of fibre-reinforced anisotropic materials. Journal of Engineering Mathematics, (https://doi.org/10.1007/s10665-017-9940-8), 2017.

[17] C. J. Cyron and J. D. Humphrey. Growth and remodeling of load-bearing biological soft tissues. Meccanica, 52:645-664, 2017.

[18] M. S. Sacks. Incorporation of experimentally-derived fiber orientation into a structural constitutive model for planar collagenous tissues. J. Biomech. Eng. - Trans. ASME, 125:280$287,2003$.

[19] S. Federico and W. Herzog. Towards an analytical model of soft biological tissues. Journal of Biomechanics, 41:3309-3313, 2008.

[20] A. Goriely. The mathematics and mechanics of biological growth. Springer, 2017.

[21] A. Pandolfi, A. Gizzi, and M. Vasta. Coupled electro-mechanical models of fiber-distributed active tissues. J. Biomech., DOI: 10.1016/j.jbiomech.2016.01.038, 2016.

[22] F. Maceri, M. Marino, and G. Vairo. A unified multiscale mechanical model for soft collagenous tissues with regular fiber arrangement. Journal of Biomechanics, 43:355-363, 2010 .

[23] Y. Lanir. Constitutive equations for fibrous connective tissues. Journal of Biomechanics, 16:1-12, 1983.

[24] T. C. Gasser, R. W. Ogden, and G. A. Holzapfel. Hyperelastic modeling of arterial layers with distributed collagen fibre orientations. Journal of the Royal Society Interface, 3:15-35, 2006 . 
[25] S. Federico and T. C. Gasser. Nonlinear elasticity of biological tissues with statistical fibre orientation. Journal of the Royal Society Interface, 7:955-966, 2010.

[26] A. Pandolfi and M. Vasta. Fiber distributed hyperelastic modeling of biological tissues. Mechanics of Materials, 44:151-162, 2012.

[27] K. Hashlamoun, A. Grillo, and S. Federico. Efficient evaluation of the material response of tissues reinforced by statistically oriented fibres. ZAMP, 67:113, 2016.

[28] E. M. Arruda and M. C. Boyce. A three-dimensional constitutive model for the large stretch behavior of rubber elastic materials. Journal of the Mechanics and Physics of Solids, 41:389$412,1993$.

[29] M. A. Zulliger, A. Rachev, and N. Stergiopulos. A constitutive formulation of arterial mechanics including vascular smooth muscle tone. American Journal of Physiology - Heart Circulation Physiology, 287:H1335-H1343, 2004.

[30] A. Gizzi, A. Pandolfi, and M. Vasta. A generalized statistical approach for modeling fiberreinforced materials. Journal of Engineering Mathematics, 108:211-226, 2018.

[31] A. Gizzi, A. Pandolfi, and M. Vasta. Statistical characterization of the anisotropic strain energy in soft materials with distributed fibers. Mechanics of Materials, 92:119-138, 2016.

[32] K. Li, R. W. Ogden, and G. A. Holzapfel. Computational method for excluding fibers under compression in modeling soft fibrous solids. Eur. J. Mech. A, Solids, 57(178-193), 2016.

[33] M. Vasta, A. Gizzi, and A. Pandolfi. On three- and two-dimensional fibers distributed models of biological tissues. Probabilistic Engineering Mechanics, 37:170-179, 2014.

[34] G. A. Holzapfel and R. W. Ogden. On fiber dispersion models: Exclusion of compressed fibers and spurious model comparisons. J. Elast., (DOI 10.1007/s10659-016-9605-2), 2016.

[35] G. A. Holzapfel and R. W. Ogden. On the tension-compression switch in soft fibrous solids. European Journal of Mechanics A/Solids, 49:561-569, 2015.

[36] G. Casella and R. Berger. Statistical inference. Brooks/Cole, 2008.

[37] R. W. Ogden. Non-linear Elastic Deformations. Dover Publications, New York, 1997. 


\section{A Application to a uniaxial tensile deformation}

We consider an incompressible material characterized by a transversely isotropic distribution of fibers with mean direction $\mathbf{M}=\mathbf{e}_{3}$ and apply a homogeneous uniaxial deformation $\lambda_{3}=\lambda$ :

$$
[\mathbf{M}]=[0,0,1]^{T}, \quad[\mathbf{F}]=\operatorname{diag}\left[\lambda^{-1 / 2}, \lambda^{-1 / 2}, \lambda\right] .
$$

A generic unit vector $\mathbf{N}$ with components

$$
[\mathbf{N}]=[\sin (\Theta) \cos (\Phi), \sin (\Theta) \sin (\Phi), \cos (\Theta)]^{T}
$$

is inclined of an angle $\Theta$ with respect to $\mathbf{M}$. This particular choice allows to write the fourth pseudo-invariant as a function of the sole variable $\Theta$ as

$$
\mathrm{I}_{4}(\Theta)=\lambda^{2}+\left(\frac{1}{\lambda}-\lambda^{2}\right) \sin (\Theta)^{2}=\frac{1}{\lambda}+\frac{\lambda^{3}-1}{\lambda} \cos (\Theta)^{2} .
$$

The local stability condition for Eq. (A.3) has been explicitly derived in [31]. For the particular case $\lambda>1$ the stability condition reads

$$
0 \leq \Theta \leq \arccos \frac{1}{1+\lambda+\lambda^{2}} \cup \pi-\arccos \frac{1}{1+\lambda+\lambda^{2}} \leq \Theta \leq \pi, \quad \text { for } \quad \Theta \in[0,2 \pi]
$$

Because of the symmetry of the arccos function, we can define the PDF in the reduced range $\Theta \in[-\pi / 2, \pi / 2]$. Under this condition, Eq. (A.4) reduces to

$$
-\arccos \frac{1}{\sqrt{1+\lambda+\lambda^{2}}} \leq \Theta \leq \arccos \frac{1}{\sqrt{1+\lambda+\lambda^{2}}} .
$$

The close-form of $\rho_{\mathrm{I}_{4}}\left(I_{4}\right)$ can be readily derived, i.e.:

$$
\rho_{\mathrm{I}_{4}}\left(I_{4}\right)=\frac{1}{N_{\mathrm{I}_{4}}} \frac{1}{\sqrt{\lambda^{3}-1} \sqrt{\lambda I_{4}-1}} \exp \left[b\left(2 \frac{\lambda I_{4}-1}{\lambda^{3}-1}\right)-1\right] .
$$

An useful application of the closed form statistics of $\mathrm{I}_{4}$ is the following.

When dealing with the generalized von Mises PDF, $\rho_{\ominus, \Phi}(\theta, \phi)$, it is necessary to evaluate the averaged generalized structure tensor $\mathbf{H}$ as (cf. [30])

$$
\mathbf{H}=\int_{0}^{\pi} \int_{-\pi / 2}^{\pi / 2} \rho_{\Theta, \Phi}(\theta, \phi) \mathbf{A} \sin \theta d \theta d \phi .
$$

When the generic orientation $\mathbf{N}$ defined in Eq. (2) is used, the six independent components 


$$
\begin{aligned}
& \alpha_{11}=\int_{0}^{\pi} \int_{-\pi / 2}^{\pi / 2} \rho_{\Theta, \Phi}(\theta, \phi) \sin ^{3} \theta \cos ^{2} \phi d \theta d \phi \\
& \alpha_{12}=\int_{0}^{\pi} \int_{-\pi / 2}^{\pi / 2} \rho_{\Theta, \Phi}(\theta, \phi) \sin ^{3} \theta \sin \phi \cos \phi d \theta d \phi \\
& \alpha_{22}=\int_{0}^{\pi} \int_{-\pi / 2}^{\pi / 2} \rho_{\Theta, \Phi}(\theta, \phi) \sin ^{3} \theta \sin ^{2} \phi d \theta d \phi \\
& \alpha_{23}=\int_{0}^{\pi} \int_{-\pi / 2}^{\pi / 2} \rho_{\Theta, \Phi}(\theta, \phi) \sin ^{2} \theta \cos \theta \sin \phi d \theta d \phi \\
& \alpha_{33}=\int_{0}^{\pi} \int_{-\pi / 2}^{\pi / 2} \rho_{\Theta, \Phi}(\theta, \phi) \cos ^{2} \theta \sin \theta d \theta d \phi \\
& \alpha_{13}=\int_{0}^{\pi} \int_{-\pi / 2}^{\pi / 2} \rho_{\Theta, \Phi}(\theta, \phi) \sin ^{2} \theta \cos \theta \cos \phi d \theta d \phi
\end{aligned}
$$

When the model specializes to transversely isotropic fiber distributions, i. e., $\rho_{\Theta, \Phi}(\theta, \phi)=$ $\rho_{\Theta}(\theta) \rho_{\Phi}(\phi)=\rho_{\Theta}(\theta) / 2 \pi$, it is possible to assume the direction $\mathbf{V}_{3}$ as preferred direction $\mathbf{a}_{0}$, cf. [24]. In this case the tensor $\mathbf{H}$ assumes the well known diagonal form $\mathbf{H}_{\mathrm{o}}$, where

$$
\alpha_{11}=\alpha_{22}=\kappa, \quad \alpha_{33}=1-2 \kappa, \quad \alpha_{12}=\alpha_{13}=\alpha_{23}=0
$$

with

$$
\kappa=\frac{1}{4} \int_{0}^{\pi} \rho_{\Theta}(\theta) \sin ^{3} \theta d \theta
$$

Similarly, for the variance model proposed in [26], it is necessary to introduce an additional coefficient $\hat{\kappa}$ defined as

$$
\hat{\kappa}=\frac{1}{16} \int_{0}^{\pi} \rho_{\Theta}(\theta) \sin ^{5} \theta d \theta
$$

Remark To provide the applicability of our generalized approach in the case of transversely isotropic fiber distributions, it is important to denote the preferred direction $\mathbf{a}_{0}$ in a different way with respect to [24]. In fact, the particular spectral decomposition used in this approach requires to name the principal stretches in decreasing order $\lambda_{1}>\lambda_{2}>\lambda_{3}$. Thus, to model the application of a uniaxial stretch in the preferred direction of the fibers, the averaged generalized second order structural tensor $\mathbf{H}$ must be derived by considering the distribution of the fibers around the principal direction $\mathbf{V}_{1}$. In a transversely isotropic framework, this means considering a distribution uniform with respect to the angle $\Theta$ and a von Mises-like distribution for the angle $\Phi$. These assumptions allow the derivation of the generalized structure tensor $\mathbf{H}=\mathbf{H}_{\mathrm{G}}$ and of their integral coefficients.

According to (A.1), the closed-form statistics of $\mathrm{I}_{4}$ allow to calculate directly the integral coefficients $\kappa$ and $\hat{\kappa}$. In particular, for transversely isotropic distributions the coefficients 
are given by

$$
\begin{aligned}
\kappa & =\frac{\lambda\left(I_{4}^{*}-\lambda^{2}\right)}{2\left(1-\lambda^{3}\right)}, \\
\hat{\kappa} & =\frac{\lambda^{2}\left(\lambda^{4}-\lambda^{3} \sigma_{I_{4}}^{2}-I_{4}^{*} \lambda^{2}+\sigma_{I_{4}}^{2}\right)}{8\left(\lambda^{3}-1\right)^{2}} .
\end{aligned}
$$

The functional dependence of the integral coefficients $\kappa$ and $\hat{\kappa}$ on $I_{4}^{*}$ and $\sigma_{I_{4}}^{2}$, respectively, is visualized in Fig. A.1 for a given value of $\lambda=1.2$. Plots highlight a linear relation between $\kappa$ and $I_{4}^{*}$ in the range of admissible values $[0.333,0]$, as well as between $\hat{\kappa}$ and $\sigma_{I_{4}}^{2}$. Note that $\hat{\kappa}$ assumes negative values for large $\sigma_{I_{4}}^{2}$.

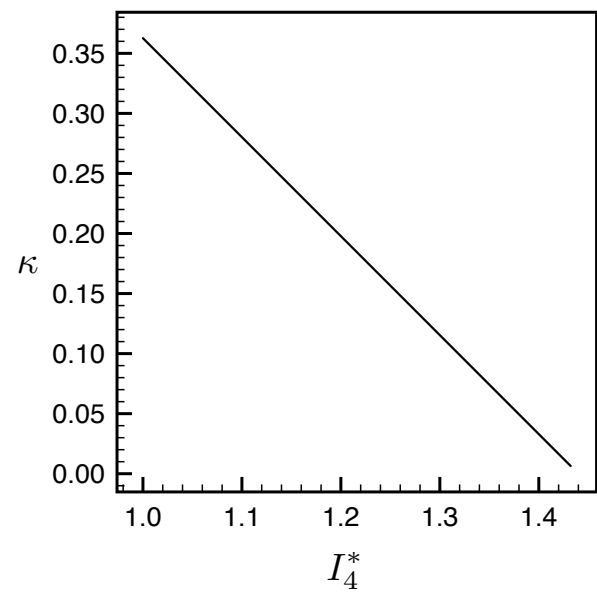

(a)

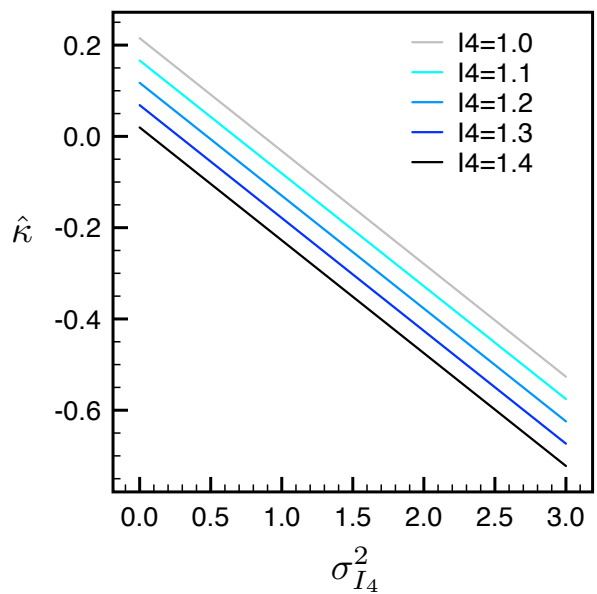

(b)

Fig. A.1. Functional dependence of $\kappa$ on $I_{4}^{*}$ and $\hat{\kappa}$ on $\sigma_{I_{4}}^{2}$, cf. Eqs. (A.8a)-(A.8b), respectively, for $\lambda=1.2$ and different values of $I_{4}^{*}$.

Remark For transversally isotropic distributions, the $\kappa$ plot corresponds to the one reported in [31]. Interestingly, the derivation of $\rho_{\mathrm{I}_{4}}\left(I_{4}\right)$ allows to compute the integral coefficients of the average structure tensors $\mathbf{H}, \mathbb{H}$ by simple integration of $\rho_{\mathrm{I}_{4}}\left(I_{4}\right)$.

Remark The coefficients $\kappa$ and $\hat{\kappa}$ coincide with the original ones proposed in [24] and [26], respectively, only if $\mathbf{N}$ is oriented as in Eq. (A.2) and $\mathbf{F}$ is as in Eq. (A.1). In general, the relation can be only written in integral form. 